\title{
Decline in independence after three years and its association with dietary patterns and IADL-related factors in community- dwelling older people: an analysis by age stage and sex
}

\author{
Sayuri Kodama ${ }^{1 *}$ (D) Tanji Hoshi ${ }^{2}$ and Sugako Kurimori ${ }^{3}$
}

\begin{abstract}
Background: Few studies have shown age stage and sex differences in the association among dietary patterns and various health factors related to disability in older people. This study aimed to reveal the differences of characteristics, including several dietary patterns, associated with a decline in independence over 3 years in community-dwelling independent older people. Specifically, we examined data by age stage, for people between 65 and 75 years (earlier-stage) and people aged 75 years or above (later-stage), and sex.

Methods: We conducted a nationwide longitudinal study of 25 Japanese prefectures from 2013 to 2016; 2250 participants' complete data (1294 men and 956 women) were analyzed. Independence was evaluated based on instrumental activities of daily living $(I A D L)$ scores (maximum $=12$ ). Dietary patterns were derived from a principal component analysis of the seven food groups. Baseline IADL-related factors linked to independence 3 years later were selected. Multiple logistic regression analysis for having low independence-without a full score of IADL 3 years after baseline - was conducted, adjusted for baseline IADL scores. Finally, to compare differences among age stage and sex groups, we used Multiple-Group Path Analysis.

Results: Participants with a full IADL score 3 years later were classified as high independence (69.6\%), and those without the full score were classified as low independence (30.4\%). Only the later-stage older peoples' proportion of low independence 3 years later was significantly higher than those at baseline. A high meat frequency pattern was associated with a significantly higher risk of decline in independence 3 years later in later-stage older women. The earlier-stage older people showed that $18.5 \leq \mathrm{BMI}<25$ was associated with a lower risk, referring to $\mathrm{BMI}<18.5$. In the later-stage, exercising three or more times a week with enjoyment and fulfillment was associated with a significantly lower risk.
\end{abstract}

\footnotetext{
* Correspondence: kodama_sayuri@isc.sagami-wu.ac.jp

1 Department of Food and Nutrition Science, Sagami Women's Junior

College, 2-1-1 Bunkyo, Minami-ku, Sagamihara-shi, Kanagawa-ken 252-0383,

Japan

Full list of author information is available at the end of the article
}

(c) The Author(s). 2021, corrected publication 2021. Open Access This article is licensed under a Creative Commons Attribution 4.0 International License, which permits use, sharing, adaptation, distribution and reproduction in any medium or format, as long as you give appropriate credit to the original author(s) and the source, provide a link to the Creative Commons licence, and indicate if changes were made. The images or other third party material in this article are included in the article's Creative Commons licence, unless indicated otherwise in a credit line to the material. If material is not included in the article's Creative Commons licence and your intended use is not permitted by statutory regulation or exceeds the permitted use, you will need to obtain permission directly from the copyright holder. To view a copy of this licence, visit http://creativecommons.org/ licenses/by/4.0/. The Creative Commons Public Domain Dedication waiver (http://creativecommons.org/publicdomain/zero/1. 0/) applies to the data made available in this article, unless otherwise stated in a credit line to the data. 
Conclusions: The IADL disability in older people aged 75 and over showed a rapid change. The different characteristics associated with the risk of decline in independence among age stage and sex were revealed. Targeting age stage and sex separately for community-based comprehensive supportive strategies would be necessary for a long life globally.

Keywords: Independence, Dietary pattern, Instrumental activities of daily living (IADL), Cohort study

\section{Background}

Although the Japanese overall life expectancy is the longest globally, the difference between healthy life expectancy and overall life expectancy was 8.84 years for men and 12.35 years for women in 2016 [1]. Older people are generally defined as those aged 65 years or older. The risk of requiring nursing care increases at 75 years or older (later-stage older people). It has been reported that those aged $\geq 75$ years have multiple diseases that precede disability, such as cerebrovascular disorder, cancer, arthropathy, and fracture [2]. In Japan, the Medical Care System for later-stage older people is separate from the National Health Insurance for those aged $<75$. Laterstage older people have a duty of $10 \%$ copayment, and those aged 70 to 74 years have a $20 \%$ copayment, except for those with income comparable to the current workforce (30\% copayment) [3]. Much more effort is needed to reduce medical costs for older people.

In 2025, the baby boomer generation in Japan will be 75 years or older, and the proportion of later-stage older people will increase. What is required for this superaged society is "community-oriented medical care," emphasizing quality of life for older people [4]. Creating a community-based system that can provide integrated living support, medical and health services, and long-term care prevention, especially for later-stage older people, is an urgent issue [5]. Nonetheless, effectively integrated support methods, understanding the effectiveness and priority of targeting support by age stage and sex separately in older people, have not yet been fully established. The supportive strategies for older people's disability prevention, as it stands now, have been primarily targeted at older people of all ages, without considering the needs of their age groups, separately [6].

Diet and nutrition are highly associated with the risk of cognitive decline and dementia in older people. Dietary patterns, which are examined by combinations of foods, have many advantages over individual nutrients or foods [7]. A Mediterranean diet, dietary approaches to stop hypertension (DASH) diet [8], and other healthy dietary patterns derived both a priori (e.g., Healthy Eating Index) [9] and a posteriori (e.g., principal component analysis [PCA], cluster analysis) [10] were shown to be associated with less cognitive decline and/or a reduced risk of dementia. A Japanese-type dietary pattern with low consumption of animal protein, such as beef and pork, and high rice consumption, miso soup, and fish was also related to a healthy life expectancy with independence [11].

There might be age stage and sex differences in the relationship between dietary patterns and older people's health. A Canadian cohort study conducted on older people aged 67 to 84 showed that adhering to unhealthy Western dietary patterns was related to poorer baseline cognitive function in men but not in women [12]. Another Canadian study conducted for those aged 85 years or older (super-seniors: SS) reported that "Western dietary pattern," characterized by high-fat food including red meat, was associated with increased odds of being an SS. However, "nutrient-rich" dietary patterns did not remain significant in an adjusted model [13].

Additionally, health status, including older people's diet quality, might be determined by the complex influence of various factors [4, 14-17]. For example, social participation is strongly associated with older people, resulting in reduced prevention [14]. Hoshi [15] clarified that there is an indirect relationship between socioeconomic status (SES) and healthy life expectancy mediated by the effects of environmental quality, mental, physical, and social health. Moreover, dietary patterns, which have a strong impact on older people's independence, might also be determined by multiple factors $[16,17]$. The authors in this study revealed that the associations between dietary diversity patterns and subjective health were mediated by mental and emotional well-being factors among older Japanese farm villages [16]. It has also been reported that higher education levels and favorable lifestyle are predictors of increased diet quality [17]. Therefore, considering other factors related to older people's individual and social factors, such as physical activity, subjective health, cognitive function, and community involvement, it is crucial to investigate effective community-based integrated support focusing on dietary patterns. However, few studies have shown the comprehensive association of dietary patterns, especially for those aged 75 years or older, separately. Furthermore, it was indicated that it might be useful to investigate the impact of several dietary patterns simultaneously to elucidate relationships with health outcomes [7].

Therefore, we conducted a nationwide longitudinal survey of community-dwelling older people in Japan to assess respondents' instrumental activities of daily living 
(IADL) at baseline and 3 years later, and evaluated changes in the degree of their independence. We comprehensively analyzed the association between a decline in independence over 3 years, several dietary patterns, and IADL-related factors in community-dwelling independent older people, as analyzed by the age stage of two groups: people aged 65-75 years (earlier-stage older people) and people aged 75 years or older (later-stage older people) and sex. This study aimed to reveal the differences of characteristics of the factors, including several dietary patterns, associated with a decline in independence over 3 years in community-dwelling independent older people. Specifically, we examined data by age stage, for people between 65 and 75 years (earlierstage) and people aged 75 years or above (later-stage), and sex. The findings would contribute to providing evidence on the future supportive strategies for preventing the decline in older people's independence, targeting age stage and sex separately.

\section{Methods}

\section{Study participants}

This longitudinal survey, which was planned and conducted in collaboration with the Foundation of Social Development for Senior Citizens (FSDSC, Tokyo) and Tokyo Metropolitan University, involved 25 prefectures in Japan. In 2013, a baseline survey of 9508 residents, who had taken part in healthy longevity events carried out by the FSDSC, was conducted (response rate: 45.7\%). The FSDSC conducts various projects, e.g., The National Health and Welfare Festival "Nenrin-pic," since 1988, and research to promote well-being and healthy longevity support among older people. The FSDSC staff sent a questionnaire to the participants in person or by mail. A follow-up survey using the same questionnaire was conducted 3 years later. It involved 3990 respondents from the baseline survey who consented to cooperate in the second and subsequent surveys (response rate: $92.6 \%$ ). A previous study described the details of this survey [18].

From the 3693 valid respondents in both surveys, we excluded participants younger than 65 years $(n=510)$ and those who died $(n=35)$. We also excluded missing data $(n=866)$ for age $(n=24)$, the questions regarding IADL index both at baseline $(n=198)$ and 3 years later $(n=151)$, those related to dietary intake frequency $(n=$ $341)$, and IADL-related factors $(n=152)$. The participants' independence was evaluated using the IADL score [19]. In the current study "disability" was operationally defined as participants with an IADL score (12 full scores) of less than 9, as described in detail below. After excluding 32 individuals, we analyzed the responses of 2250 people (men: 1294, women: 956) who were identified as independent.

\section{Evaluation of independence and classification of study participants}

We used the 13-item Tokyo Metropolitan Institute of Gerontology Index of Competence (TMIG-IC) to assess independence. It evaluates multiple dimensions of IADL, including intellectual and social ADL, among older people [19]. In this study, the TMIG-IC was modified to contain 12 items (Table 1). We converted two questions, "Do you read newspapers?" and "Do you read books or magazines?" into a single item, "Do you read newspapers or books?" The responses of "Yes" and "No" were scored as 1 and 0 , respectively, and were summated to obtain each respondent's IADL score. The higher the IADL score (up to 12 points), the higher an individual's degree of independence.

Since no cutoff point has been reported for the 13item TMIG-IC [19], we referred to Fujiwara's study, which defined participants with scores above 10 IADL at baseline to be almost independent in daily living [20]. As mentioned above, we converted two questions into a single question and modified the scale to contain 12 items. Therefore, to decide on the cutoff point for disability in this study's participants, we examined the relationship with the response rate of questions for each IADL evaluation item for the participants with IADL scores of 8, 9, and 10 points (chi-square test). The evaluation items that showed statistically significant relationships were Instrumental Self-Maintenance (5 full scores) and Social Role ( 4 of full score). The percentage of participants who were able to do all items of the Instrumental SelfMaintenance questions was significantly lower in the participants who had IADL scores of 8 points (33.3\%) than those of 9 points (58.7\%) and 10 points (72.5\%). Besides, no one had a full score on the Social Role questions in the 8-point participants. Their score was significantly less than those with 9 points (7.9\%) and 10 points $(8.2 \%)$. From the results of these tests, those with 8 points were considered to have a low degree of independence. Therefore, in this study, disability was operationally defined as participants with IADL scores lower than 9 with a modified 12 -item index. The participants' IADL scores 3 years after the baseline were calculated. The group was then divided into two groups: the full score formed the high independence group, and the other, without a full score, formed the low independence group.

\section{Evaluation of dietary intake frequency}

Dietary habits were collected using the frequency of consumption of food groups in the cooking units per week. Seven food groups (meat dishes, soy products, eggs/egg dishes, bluefish, dairy products, fruits, and vegetable dishes) were examined. The frequent consumption of 
Table 1 Instrumental ADL: IADL questionnaire

\begin{tabular}{ll}
\hline No. & Questions \\
\hline Instrumental Self-Maintenance & \\
$\mathbf{1}$ & Can you use public transportation (bus or train) by yourself? \\
$\mathbf{3}$ & Are you able to shop for daily necessities by yourself? \\
$\mathbf{4}$ & Are you able to prepare meals by yourself? \\
$\mathbf{5}$ & Are you able to pay bills? \\
Intellectual Activity & Can you handle your own banking? \\
$\mathbf{6}$ & \\
$\mathbf{7}$ & Are you able to fill out forms for your pension? \\
$\mathbf{8}$ & Do you read newspapers or books? \\
$\mathbf{9}$ & Are you interested in news stories or programs dealing with health? \\
$\mathbf{1 0}$ & \\
$\mathbf{1 1}$ & Do you visit the homes of friends? \\
\hline
\end{tabular}

The answer is either "yes" or "no." Each answer will be given 1 point for "yes"

Referring to the TMIG index of Competence from Koyano W, et al. [19]

bluefish, which is rich in n-3 fatty acids, is related to the maintenance of older people's cognitive functions.

Scoring was based on an ordinal scale: 5 points for "every day," 4 points for " $5-6$ days a week," 3 points for "3-4 days a week," 2 points for "1-2 days a week," and 1 point for "never." We examined whether these scores were adequate predictors of participants' independence 3 years after baseline. The association was analyzed using the sum of all scores as the dietary diversity score-the group who had low independence 3 years after baseline was set as the dependent variable. The logistic regression analysis, adjusted for sex and age, showed that the dietary diversity scores were significantly related to the prevention of low independence 3 years after baseline (odds ratio [OR] $=0.947,95 \%$ confidence interval [CI]: 0.926-0.969). Therefore, this study found that a higher score for dietary intake frequency signaled that a respondent was more likely to maintain independence 3 years from baseline.

\section{Analysis of IADL-related factors}

The items related to IADL were selected referring to the "Questionnaire for the Elderly" [21], provided by the Japanese Ministry of Health, Labour, and Welfare, to evaluate later-stage health conditions of older people. The items used in this analysis included (1) health status, (2) psychological and mental health status, (3) weight change, (4) exercise indices related to falls, (5) smoking, and (6) social participation. (1) For the health condition, "no hospitalization over the previous year" (yes, no) was used. (2) Ordinal scale scores for subjective health $(1=$ unhealthy, 2 =somewhat unhealthy, $3=$ somewhat healthy, $4=$ excellent $)$ and life satisfaction $(1=$ not satisfied, $2=$ neither nor, $3=$ satisfied) were used to identify psychological and mental health conditions. (3) Body mass index (BMI) was calculated using self-reported height and weight and was classified into four categories: $\mathrm{BMI}<18.5,18.5 \leq \mathrm{BMI}<25,25 \leq \mathrm{BMI}<30$, and $\mathrm{BMI} \geq$ 30. (4) For exercise frequency, we used the index of "exercise frequency with enjoyment and fulfillment" [18] that we developed for a previous study for the same participants. Besides the high frequency of exercise for older people, enjoyment and fulfillment are strongly related to maintaining independence 3 years from baseline [18]. There were two questions. The first asked, "How much do you exercise or play sports?" We classified each respondent as "exercises three or more times a week $(\geq 3$ times/week)" or "exercises two or fewer times a week ( $\leq 2$ times/week)." Next, we asked the participants about their enjoyment and fulfillment. If they responded that they enjoyed their exercise, we reclassified them as "exercises three times or more per week with enjoyment and fulfillment ( $\geq 3$ times/week, E\&F)" (or not) and "exercises twice or less per week with enjoyment and fulfillment ( $\leq 2$ times/week, E\&F)" (or not). Finally, four categories were created for the exercise index. (4) We also inquired about "no fall fractures" that had occurred over the previous year (or not). (5) We asked the participants to self-report their cigarette smoking status (current, former, never). (6) The respondents' social participation was tallied through the frequency of their community and volunteer activity engagement (yes, 
sometimes, or no). We examined the above baseline items to determine if they were related to independence 3 years later, and we used them in the multivariable analysis if significant. BMI categories did not show a significant single relationship, but we used it as an adjusting factor.

\section{Other variables}

We also analyzed variables related to demographic and SES, including age, sex, the household status of living alone (yes, no), ordinal scaled scores for economic satisfaction $(1=$ not satisfied, $2=$ not very satisfied, 3 =moderately satisfied, $4=$ satisfied), and salaried employees (yes, no). We used economic satisfaction as an alternative SES variable instead of annual income because older people's subjective economic status is more closely related to psychological health than annual income [22].

\section{Analysis}

Comparing the proportion of independence between baseline and 3 years later by age stage and sex groups was analyzed by conducting McNemar's test. The baseline characteristics were compared between the high- and low-independence groups after 3 years using the Mann-Whitney $U$ test for ordinal scaled scores (including dietary intake frequency). Chisquared tests were used for all the other variables. To identify dietary patterns, we performed PCA using the dietary intake frequency scores for seven types of food groups. PCA is the most common data-driven method used to derive dietary patterns. Its analytic advantage is that it results in a continuous score, maximizing statistical power to examine the relationship between diet and disease [7].

With the IADL score after 3 years as the dependent variable, two-way ANOVA was conducted for all variables to verify whether there was an effect-modification (interaction) due to the baseline age stage or sex. Multivariate logistic regression analysis was conducted to evaluate the associations among decline in independence after 3 years, dietary patterns, and IADL-related factors [23]. The principal component scores, which are uncorrelated with each other, were used to evaluate the dietary patterns. This analysis's outcome variable had low independence-without a full score of IADL-after 3 years. ORs and 95\% CIs were calculated separately for age stage and sex. The multivariate models were adjusted for IADL scores at baseline. In addition, to compare differences among age stage and sex groups, Multiple-Group Path Analysis was conducted for age stage, sex, and their combination, simultaneously [15, 24, 25]. Path Analysis is one of the methods of a causal modeling approach for Structural Equation Modeling (SEM). We examined the direct effects of baseline characteristics on low independence 3 years later. We used the same indicators examined in the multiple logistic regression analysis, adjusting for the effect of each indicator. Standardized estimates (S.E.) that compare the magnitude of the effect between groups were calculated. Then all effects were labeled in order, and a comparison of the label's pair was generated with a critical ratio (CR). CR presents a ratio associated with differences between two effects. A statistically significant difference was indicated by a $\mathrm{CR}> \pm 1.96: P<0.05, \mathrm{CR}> \pm 2.23: P<0.01$, and $\mathrm{CR}> \pm 2.58: P<0.001$.

The statistical analyses were performed using SPSS Statistics 24.0. Multiple-Group Analysis was conducted using Amos statistical software 23.0 for Windows. Statistical significance was set at $P<0.05$.

\section{Results}

IADL score of baseline and 3 years later

The study participants were 1294 men (57.5\%) and 956 women (42.5\%) with a total of 2250 independent older people. The baseline age composition was 1545 (68.7\%) earlier-stage older people (men: $863,55.9 \%$; women: 682 , $44.1 \%)$ and $705(31.3 \%)$ later-stage older people (men: 431, 61.1\%; women: 274, 38.9\%). The average age was $72.32 \pm 5.22$ years (men: $72.56 \pm 5.43$ years, women: $72.01 \pm 4.92$ years).

The mean IADL score 3 years after baseline was $11.48 \pm 1.06$, and its median was 12 full scores. The distribution of the IADL score 3 years later is shown in Fig. 1. Participants with a score of 0 were admitted to a medical institution. Participants with a full score of 12 points 3 years after baseline, who were completely independent, were classified as "high independence" $(n=1565,69.6 \%)$. The others without a full score of IADL, who were not completely independent, were classified as "low independence" $(n=685,30.4 \%)$. The proportion of low independence respondents 3 years after baseline was $37.5 \%$ for men, $20.9 \%$ for women, $28.3 \%$ for earlier-stage older people, and $35.2 \%$ for later-stage older people. Of the low independence participants, $67.0 \%$ had no change 3 years from baseline, and $33.0 \%$ changed to high independence. Whereas $17.2 \%$ of the high independence group changed to low independence, and $82.8 \%$ maintained their status 3 years later.

Comparing the proportion of high and low independence between baseline and 3 years later by age stage and sex in the two groups, only the later-stage older peoples' results were significantly different (Fig. 2). It was shown that the proportion of low independence 3 years later was higher than those at baseline (later-stage men: baseline 30.6\%, 3 years later 39.2\%; later-stage women: 19.0, 28.8\%). 


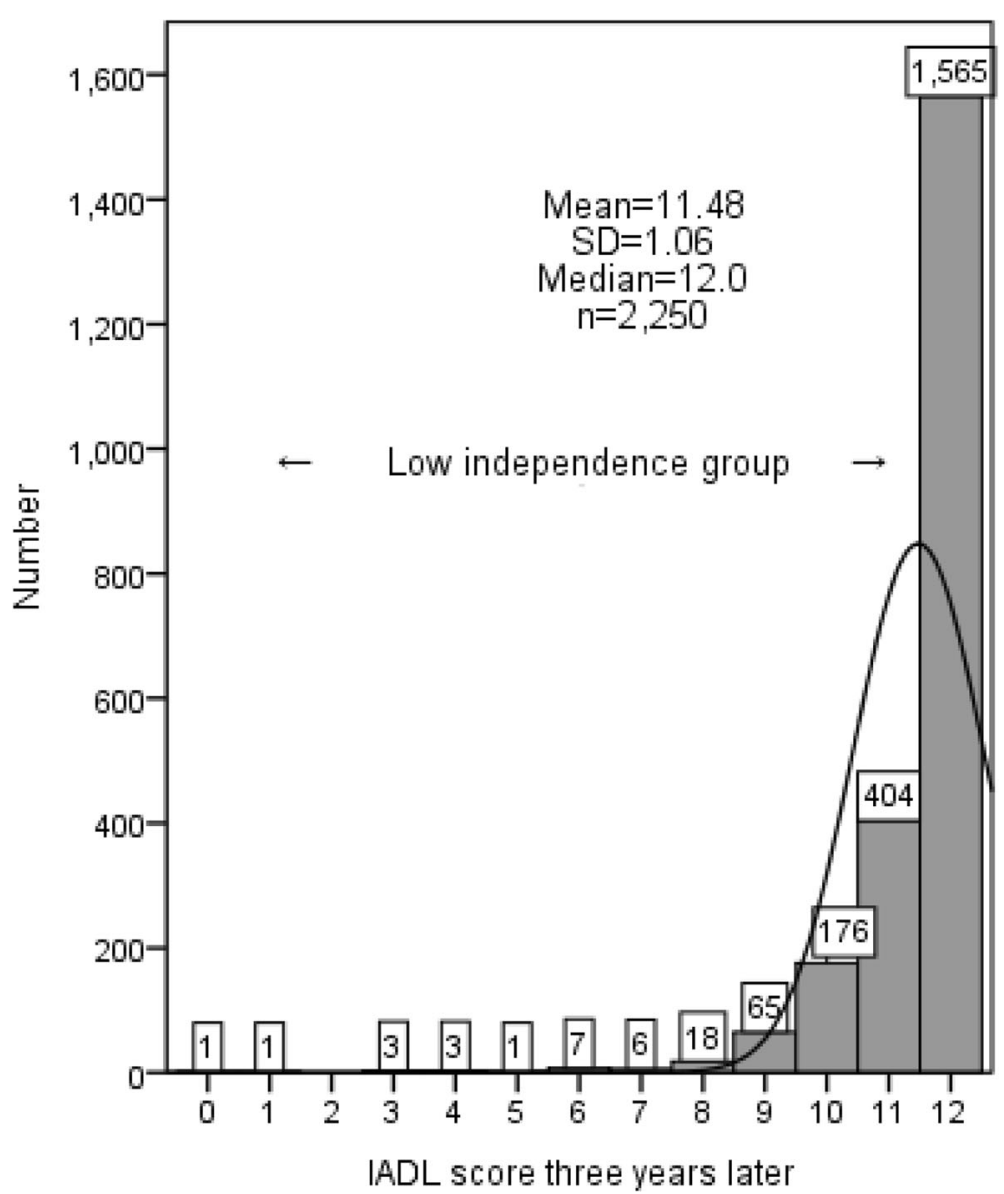

Fig. 1 Distribution of the IADL score 3 years later. IADL, Instrumental Activities of Daily Living

\section{Relationship between baseline-related factors and independence 3 years later}

Table 2 shows the relationship between the baseline values and independence 3 years later. It compares the high independence groups' results with the low independence group. The analysis was performed for each age stage. The main item with which only earlier-stage older people showed a significant relationship was smoking. The percentage of those who had never smoked in the high independence group 3 years after baseline was the highest at $77.8 \%$. The proportion of those who smoked and reached low independence was $44.4 \%$, the highest among the low independence group.

Having salaried employment was one of the main items in which only later-stage older people showed a significant relationship. The percentage of salaried employees who were among the high independence group 3 years later was the highest at $72.2 \%$. Contrastingly, the proportion of those without a low independence salary reached $36.5 \%$, the highest among the low independence respondents. The results of the exercise frequency of the later-stage older people showed that the percentage of those who exercised $\geq 3$ times/week with E\&F and were identified as having high independence 3 years after baseline was the highest at $69.1 \%$. Conversely, the proportion of those who exercised 2 times $\geq$ a week without E\&F and had low independence was $45.0 \%$, the highest among the low independence respondents.

\section{Relationship between dietary intake frequency and independence 3 years later}

Table 3 shows the relationship between baseline dietary intake frequency and independence 3 years later. It compares the high independence groups' results with those of the low independence group. Overall, the high independence group was significantly higher in each food group's intake frequency than the low independence group. However, the percentage of those who ate meat 


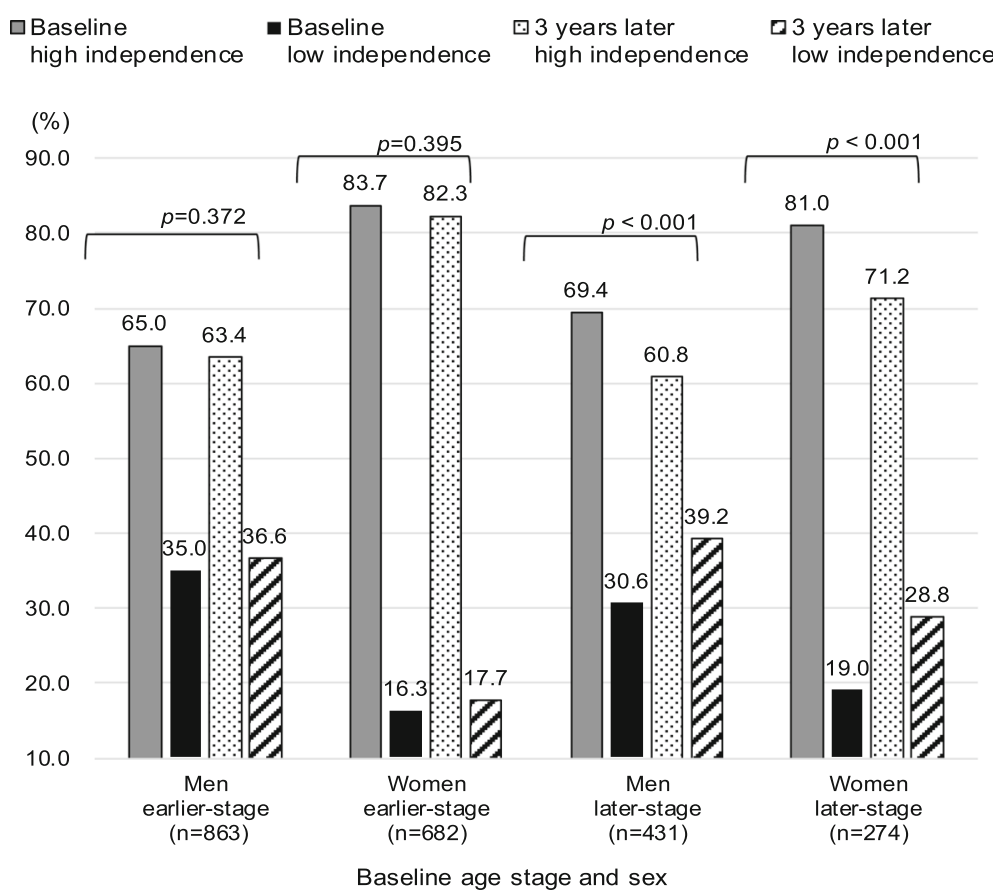

Fig. 2 Comparison of proportion of independence between baseline and 3 years later

every day and reached low independence 3 years later was high among the low independence group (overall result: $32.7 \%)$. Further, it did not differ significantly from those who ate meat $1-2$ days a week (32.7\%) or did not eat meat $(32.8 \%)$.

\section{Dietary patterns}

PCA identified three components that explained 59.4\% of the total variance (Table 4). The first component was characterized by dietary variety that showed that all food groups had a strong relationship with a high frequency of eating throughout the week. It was described as "dietary diversity." The second pattern was a negative relationship for vegetable, fruit, and dairy products, which was called "low fruit, vegetable, and dairy product frequency." The third pattern had a negative relationship with soy products and bluefish and a strong positive relationship with a high frequency of meat dishes during the week (principal component loading: 0.660); it was called "high meat frequency."

\section{Comprehensive analysis for having low independence 3 years from baseline}

At first, we tested all variables to determine whether there was an effect-modification (interaction) due to the baseline age stage or sex, with the IADL score after 3 years as the dependent variable. Baseline indicators that significantly interacted with the baseline age groups were frequency of soy products $(\mathrm{F}=2.61, \quad P=0.034)$, vegetarian food $(\mathrm{F}=4.66, P=0.003)$, financial satisfaction $(\mathrm{F}=4.66, \quad P=0.006)$, smoking Habits $(\mathrm{F}=4.47, \quad P=$ $0.012)$, and subjective health $(\mathrm{F}=5.41, P=0.001)$. We confirmed that gender interactions were not significantly associated with any variables.

Next, we conducted a multiple logistic regression analysis for having low independence 3 years after baseline. It was analyzed by age stage and sex separately using indices showing significant relationships with independence 3 years later, which showed no correlation coefficient of $\geq 0.5$ between the indices.

First, we conducted the unadjusted single logistic regression analysis for being low independent 3 years after baseline with each individual dietary pattern derived from the PCA (Table 5). In earlier-stage older people, dietary diversity was associated with a significant lower risk of decline in independence 3 years after baseline regardless of sex (men: $\mathrm{OR}=0.82,95 \%$ CI: 0.72-0.94, women: $\mathrm{OR}=0.75,95 \%$ CI: $0.60-0.94$ ). While high meat frequency was associated with a significant higher risk of decline in independence (men: $\mathrm{OR}=1.16,95 \%$ CI: $1.01-1.35$, women: $\mathrm{OR}=1.22$, 95\% CI: 1.00-1.48). On the other hand, in later-stage older people, a significantly lower risk of decline in independence for dietary diversity was shown only in men $(\mathrm{OR}=0.75,95 \% \mathrm{CI}: 0.62-0.91)$, and the significanthigher risk of decline in independence for high meat frequency was observed only in women $(\mathrm{OR}=1.64$, 95\% CI: 1.24-2.17). 


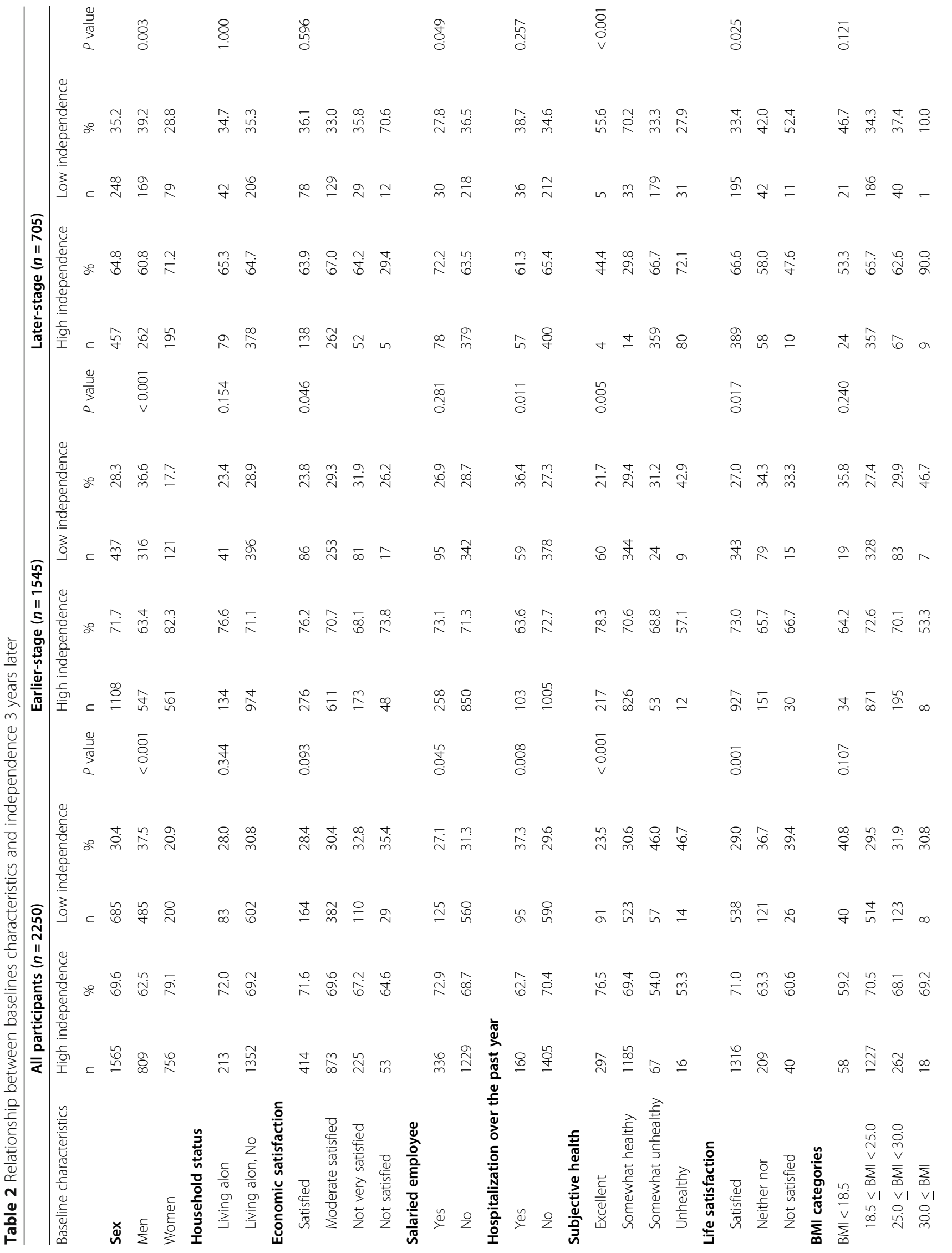




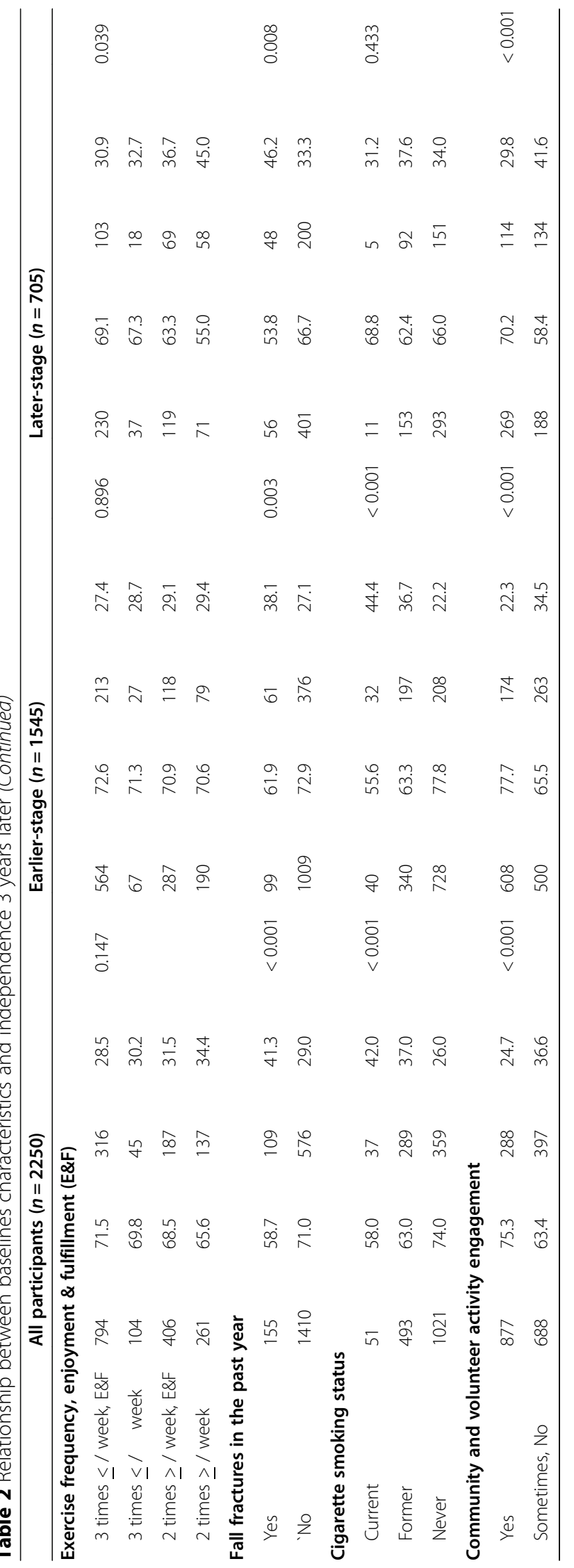


Table 3 Relationship between dietary intake frequency and independence 3 years later

\begin{tabular}{|c|c|c|c|c|c|c|c|c|}
\hline \multirow{3}{*}{ Baseline characteristics } & \multicolumn{3}{|c|}{ All participants $(n=2250)$} & \multicolumn{2}{|c|}{ Earlier-stage $(n=1545)$} & & \multicolumn{2}{|c|}{ Later-stage $(n=705)$} \\
\hline & $\begin{array}{l}\text { High } \\
\text { independence }\end{array}$ & $\begin{array}{l}\text { Low } \\
\text { independence }\end{array}$ & & $\begin{array}{l}\text { High } \\
\text { independence }\end{array}$ & $\begin{array}{l}\text { Low } \\
\text { independence }\end{array}$ & & $\begin{array}{l}\text { High } \\
\text { independence }\end{array}$ & $\begin{array}{l}\text { Low } \\
\text { independence }\end{array}$ \\
\hline & $\%$ & $\%$ & $P$ value & $\%$ & $\%$ & $P$ value & $\%$ & $\%$ \\
\hline
\end{tabular}

\section{Protein foods}

\section{Meat dishes}

Every day

5-6 days a week

$\begin{array}{llll}72 & 67.3 & 35 & 32.7 \\ 151 & 69.6 & 66 & 30.4 \\ 606 & 72.7 & 227 & 27.3 \\ 693 & 67.3 & 336 & 32.7 \\ 43 & 67.2 & 21 & 32.8\end{array}$

$\begin{array}{ccccc}0.096 & 49 & 70.0 & 21 & 30.0 \\ & 101 & 71.1 & 41 & 28.9 \\ 436 & 74.8 & 147 & 25.2 \\ 495 & 69.8 & 214 & 30.2 \\ 27 & 65.9 & 14 & 34.1\end{array}$

$\begin{array}{lllll}0.158 & 23 & 70.0 & 14 & 30.0 \\ & 50 & 71.1 & 25 & 28.9 \\ 170 & 74.8 & 80 & 25.2 \\ 198 & 69.8 & 122 & 30.2 \\ 16 & 65.9 & 7 & 34.1\end{array}$

Soy products

\section{Every day}

5-6 days a week

3-4 days a week

1-2 days a week

Never

\section{Eggs/egg dishes}

$\begin{array}{lllll}\text { Every day } & 342 & 70.5 & 143 & 29.5 \\ \text { 5-6 days a week } & 226 & 73.1 & 83 & 26.9 \\ \text { 3-4 days a week } & 553 & 70.4 & 233 & 29.6 \\ \text { 1-2 days a week } & 417 & 66.7 & 208 & 33.3 \\ \text { Never } & 27 & 60.0 & 18 & 40.0\end{array}$

\section{Bluefish}

Every day

5-6 days a week

3-4 days a week

1-2 days a week

Never

Other foods

\section{Vegetable dishes}

\begin{tabular}{|c|c|c|c|c|c|c|c|c|c|c|c|c|c|}
\hline Every day & 1138 & 72.6 & 429 & 27.4 & $<0.001$ & 791 & 75.2 & 261 & 24.8 & $<0.001$ & 347 & 74.9 & 168 \\
\hline 5-6 days a week & 253 & 64.1 & 142 & 35.9 & & 182 & 62.8 & 108 & 37.2 & & 71 & 74.1 & 34 \\
\hline 3-4 days a week & 140 & 61.4 & 88 & 38.6 & & 110 & 69.2 & 49 & 30.8 & & 30 & 65.6 & 39 \\
\hline 1-2 days a week & 34 & 57.6 & 25 & 42.4 & & 25 & 56.8 & 19 & 43.2 & & 9 & 62.7 & 6 \\
\hline Never & 0 & 0.0 & 1 & \#\#\#\# & & 0 & 0.0 & 0 & 0.0 & & 0 & 66.7 & 1 \\
\hline
\end{tabular}

\section{Fluits}

$\begin{array}{lllll}\text { Every day } & 932 & 72.2 & 359 & 27.8 \\ \text { 5-6 days a week } & 243 & 71.1 & 99 & 28.9 \\ \text { 3-4 days a week } & 247 & 64.7 & 135 & 35.3 \\ \text { 1-2 days a week } & 133 & 60.7 & 86 & 39.3 \\ \text { Never } & 10 & 62.5 & 6 & 37.5\end{array}$

\section{Dairy products}

Every day

$\begin{array}{llll}950 & 69.9 & 409 & 30.1\end{array}$

5-6 days a week $\begin{array}{llll}80 & 80.0 & 20 & 20\end{array}$

$\begin{array}{llll}192 & 73.8 & 68 & 26.2\end{array}$

$\begin{array}{llll}605 & 72.5 & 230 & 27.5\end{array}$

$\begin{array}{llll}647 & 65.4 & 342 & 34.6\end{array}$

$\begin{array}{llll}41 & 62.1 & 25 & 37.9\end{array}$

$\begin{array}{ccccc}<0.001 & 426 & 77.2 & 126 & 22.8 \\ & 185 & 75.8 & 59 & 24.2 \\ & 328 & 67.9 & 155 & 32.1 \\ & 168 & 64.9 & 91 & 35.1 \\ & 1 & 14.3 & 6 & 85.7 \\ 0.044 & 211 & 73.8 & 75 & 26.2 \\ & 166 & 75.1 & 55 & 24.9 \\ & 398 & 72.2 & 153 & 27.8 \\ & 314 & 68.9 & 142 & 31.1 \\ & 19 & 61.3 & 12 & 38.7\end{array}$

$\begin{array}{llll}120 & 73.6 & 43 & 26.4\end{array}$

$\begin{array}{llll}428 & 75.1 & 142 & 24.9\end{array}$

$\begin{array}{llll}477 & 67.3 & 232 & 32.7\end{array}$

$\begin{array}{llll}29 & 74.4 & 10 & 25.6\end{array}$

$54-84.4-10-15.6$
$120-73.6-43-26.4$

$\begin{array}{cllll}<0.001 & 216 & 67.9 & 98 & 32.1 \\ & 76 & 64.9 & 34 & 35.1 \\ & 110 & 14.3 & 56 & 85.7 \\ 50 & 73.8 & 56 & 26.2 \\ & 5 & 75.1 & 4 & 24.9 \\ 0.039 & 131 & 68.9 & 68 & 31.1 \\ & 60 & 61.3 & 28 & 38.7 \\ & 155 & 75.2 & 80 & 24.8 \\ 103 & 62.8 & 66 & 37.2 \\ 8 & 69.2 & 6 & 30.8\end{array}$

$\begin{array}{cllll}<0.001 & 26 & 73.6 & 10 & 26.4 \\ 72 & 75.1 & 25 & 24.9 \\ 177 & 67.3 & 88 & 32.7 \\ 170 & 74.4 & 110 & 25.6 \\ 12 & 77.2 & 15 & 22.8\end{array}$
9 5 1

0.272

0.001 2.8

0.358

0.002 6.2 .9

8


Table 3 Relationship between dietary intake frequency and independence 3 years later (Continued)

\begin{tabular}{|c|c|c|c|c|c|c|c|c|c|c|c|c|}
\hline \multirow[b]{2}{*}{ 3-4 days a week } & \multicolumn{4}{|c|}{ All participants $(n=2250)$} & \multicolumn{4}{|c|}{ Earlier-stage $(n=1545)$} & \multicolumn{4}{|c|}{ Later-stage $(n=705)$} \\
\hline & 207 & 73.1 & 76 & 26.9 & 156 & 73.2 & 57 & 26.8 & 51 & 63.5 & 19 & 36.5 \\
\hline $1-2$ days a week & 169 & 62.6 & 101 & 37.4 & 129 & 63.5 & 74 & 36.5 & 40 & 56.9 & 27 & 43.1 \\
\hline Never & 40 & 54.8 & 33 & 45.2 & 29 & 56.9 & 22 & 43.1 & 11 & 56.9 & 11 & 43.1 \\
\hline
\end{tabular}

Multiple adjusted logistic regression analysis models compared all participants with age stage groups were then examined (Table 6). The indices that showed association with a significantly lower risk of decline in independence 3 years later for both age stages were the IADL score at baseline (earlier-stage older people: $\mathrm{OR}=0.26$, 95\% CI: 0.21-0.32; later-stage older people: $\mathrm{OR}=0.20,95 \% \mathrm{CI}: 0.14-0.27$ ). The analysis by age group revealed that women were associated with a significantly lower risk of decline in independence 3 years later than men (women in earlier-stage: $\mathrm{OR}=$ 0.54, 95\% CI: $0.38-0.75$, women in the later-stage: $\mathrm{OR}=0.60,95 \%$ CI: $0.37-0.96$ ).

The dietary diversity was associated with a lower risk of decline in independence 3 years later in both age stages (Table 5); however, these effects were attenuated after adjusting for other dietary patterns or IADL-related factors and became insignificant (Table 6). Conversely, high meat frequency was associated with a significantly higher risk of decline in independence in earlier-stage individuals after adjusting for IADL related factors (OR = 1.19, 95\%CI: 1.04-1.36).

The earlier-stage older people showed an association with a significantly lower risk of decline in independence 3 years later for $18.5 \leq \mathrm{BMI}<25(\mathrm{OR}=0.50,95 \% \mathrm{CI}$ : $0.25-0.99$, reference group: $\mathrm{BMI}<18.5$ ). A $\mathrm{BMI} \geq 30.0$ tended to be a higher risk for decline in independence in earlier-stage older people $(\mathrm{OR}=1.30,95 \% \mathrm{CI}$ : $0.36-$
4.65), albeit a lower risk in all participants $(\mathrm{OR}=0.67$, 95\% CI: $0.24-1.86)$ and in later-stage individuals $(\mathrm{OR}=$ 0.11, 95\% CI: 0.01-1.10).

In the earlier-stage older people, no fall fractures in the previous year $(\mathrm{OR}=0.61,95 \% \mathrm{CI}: 0.41-0.92)$ and community and volunteer activity engagement $(\mathrm{OR}=$ $0.62,95 \%$ CI: $0.47-0.81$ ) were associated with a significantly lower risk of decline in independence 3 years later. Meanwhile, only the later-stage older people showed an association with a significantly lower risk for $\geq 3$ times/week with E\&F (OR $=0.54,95 \%$ CI: 0.33-0.91, reference group: $\leq 2$ times/week).

Subsequently, the analysis by age stage and sex was conducted (Table 7). The high meat frequency in the later-stage older women was associated with the highest risk of decline in independence 3 years later for all results, regardless of sex or age $(\mathrm{OR}=1.67,95 \% \mathrm{CI}$ : 1.18 2.35). The results revealed that earlier-stage older men showed an association with a higher risk of decline in independence for a $\mathrm{BMI} \geq 30$ (OR $=2.24,95 \% \mathrm{CI}$ : 0.25 19.9), albeit not significant, and with a significantly lower risk of decline in independence for community and volunteer activity engagement $(E \& F)(O R=0.49,95 \% \mathrm{CI}$ : $0.35-0.68)$. The later-stage older men showed an association with a lower risk of decline in independence for $\geq 3$ times/week with E\&F (OR = 0.46, 95\% CI: 0.24-0.88, reference group: $\leq 2$ times/week). The results of earlierstage older women showed an association with a lower

Table 4 Principal component analysis for dietary patterns

\begin{tabular}{|c|c|c|c|}
\hline & \multicolumn{3}{|c|}{ Component } \\
\hline & a. 1 & b. 2 & c. 3 \\
\hline Vegetable dishes & 0.645 & -0.317 & -0.132 \\
\hline Fluits & 0.643 & -0.434 & 0.138 \\
\hline Soy products & 0.621 & 0.120 & -0.432 \\
\hline Dairy products & 0.548 & -0.414 & 0.239 \\
\hline Eggs/egg dishes & 0.519 & 0.430 & 0.102 \\
\hline Bluefish & 0.466 & 0.472 & -0.398 \\
\hline Meat dishes & 0.404 & 0.475 & 0.660 \\
\hline Total variance $(\%)$ & 30.9 & 46.7 & 59.4 \\
\hline \multicolumn{4}{|c|}{$\begin{array}{l}\text { Dietary patterns were described as follows: } \\
\text { a. Component 1: Dietary diversity } \\
\text { b. Component 2: Low fruit, vegetable, and dairy product frequency } \\
\text { c. Component 3: High meat frequency }\end{array}$} \\
\hline
\end{tabular}


Table 5 Relationship between each individual dietary pattern and having low independence three years after baseline

\begin{tabular}{|c|c|c|c|c|c|c|}
\hline \multirow{2}{*}{$\begin{array}{l}\text { Unadjusted } \\
\text { dietary pattern }\end{array}$} & \multicolumn{3}{|c|}{ Earlier-stage } & \multicolumn{3}{|c|}{ Later-stage } \\
\hline & OR & $(95 \% \mathrm{Cl})$ & $P$ value & OR & $(95 \% \mathrm{Cl})$ & $P$ value \\
\hline \multicolumn{7}{|l|}{ All participants } \\
\hline Dietary diversity & 0.73 & $(0.65-0.81)$ & $<0.001$ & 0.74 & $(0.63-0.87)$ & $<0.001$ \\
\hline High meat frequency & 1.12 & $(0.99-1.25)$ & 0.054 & 1.22 & $(1.05-1.43)$ & 0.009 \\
\hline Low $F, V, D^{a}$ & 1.05 & $(0.94-1.17)$ & 0.431 & 0.95 & $(0.81-1.10)$ & 0.472 \\
\hline \multicolumn{7}{|l|}{ Men } \\
\hline Dietary diversity & 0.82 & $(0.72-0.94)$ & 0.004 & 0.75 & $(0.62-0.91)$ & 0.004 \\
\hline High meat frequency & 1.16 & $(1.01-1.35)$ & 0.042 & 1.09 & $(0.91-1.32)$ & 0.341 \\
\hline Low $F, V, D^{a}$ & 0.99 & $(0.87-1.15)$ & 0.994 & 0.95 & $(0.78-1.15)$ & 0.610 \\
\hline \multicolumn{7}{|l|}{ Women } \\
\hline Dietary diversity & 0.75 & $0.94)$ & 0.013 & 0.79 & $(0.58-1.07)$ & 0.125 \\
\hline High meat frequency & 1.22 & $(1.00-1.48)$ & 0.045 & 1.64 & $(1.24-2.17)$ & 0.001 \\
\hline Low $F, V, D^{a}$ & 0.97 & $(0.80-1.19)$ & 0.802 & 0.90 & $(0.70-1.17)$ & 0.440 \\
\hline
\end{tabular}

${ }^{a}$ Low F,V,D: Low fruit, vegetable, and dairy product frequency

risk of decline in independence for no fall fractures in the previous year $(\mathrm{OR}=0.42,95 \% \mathrm{CI}$ : $0.24-0.75)$.

\section{Multiple-group path analysis for testing the difference among age stage and sex}

To test the difference among groups of age stage and sex, we conducted Multiple-Group Path Analysis (i.e., analyzing multiple groups simultaneously). A Path analysis of SEM was used to examine the direct effect of 15 indicators (those in Tables 6 and 7) of baseline characteristics on low independence 3 years later. Each effect was labeled in order. For example, in the age stage and sex group, the direct effect of earlier-stage men's IADL baseline score was labeled as par_1, and the last label was par_60 for the effect of later-stages women's baseline age. S.E. and CR to the reference group are shown in Table 8. Only the results of the baseline indicators, with significant odds ratios in Tables 6 and 7, are listed. Negative S.E. indicated that older people were more likely to be at lower risk of low independence after 3 years.

The results that had significant associations are as follows. For the age stage difference, there was a significant difference in the magnitude of the effects of E\&F on low independence 3 years later in earlier-stage and laterstage individuals. The effect of community and volunteer activity engagement of men was significantly larger than women. For the age stage and sex difference, the effect of high meat frequency pattern among later-stage older women was significantly larger than among later-stage older men and earlier-stage older women. The result of E\&F of later-stage older men was significantly different from earlier-stage older women and community and volunteer activity engagement of earlier-stage older men was significantly larger than later-stage older men and earlier-stage older women.

\section{Discussion}

In providing evidence for building a comprehensive support strategy for preventing a decline in independence, this study conducted a comprehensive analysis of IADLrelated multidimensional factors, especially several dietary patterns, and a decline in independence after 3 years, by age stage of two groups and sex separately. In this study, the outcome of the analysis was set for low independence, defined as the participants without a full score for IADL. Only the later-stage older peoples' proportion of low independence after 3 years was significantly higher than those at baseline. The results suggest that later-stage older people in particular need more urgent health care support than earlier-stage people.

\section{Relationship between dietary patterns and low independence 3 years later}

Our study investigated three dietary patterns in the participants: dietary diversity, low fruit, vegetable, dairy product frequency, and high meat frequency. Usually, dietary patterns are examined separately with the outcome factor; however, there is a possibility of obtaining valuable results for accumulating helpful evidence for health care strategies [7]. In our multivariate analysis, three kinds of dietary patterns were used simultaneously, and we investigated the impact of every pattern for low 
Table 6 Multiple logistic regression analysis for having low independence 3 years after baseline by age stage

\begin{tabular}{|c|c|c|c|c|c|c|c|c|c|c|c|c|c|c|c|}
\hline \multirow[t]{3}{*}{ Baseline characteristics } & \multicolumn{5}{|c|}{ All participants } & \multicolumn{5}{|c|}{ Earlier-stage } & \multicolumn{5}{|c|}{ Later-stage } \\
\hline & \multicolumn{5}{|c|}{$(n=2250)$} & \multicolumn{5}{|c|}{$(n=1545)$} & \multicolumn{5}{|c|}{$(n=705)$} \\
\hline & OR & $(95 \% \mathrm{C}$ & & & $P$ value & OR & $(95 \%$ & & & $P$ value & OR & $(95 \%$ & & & $P$ value \\
\hline IADL score of baseline & 0.24 & $(0.20$ & - & $0.29)$ & $\begin{array}{l}< \\
0.001\end{array}$ & 0.26 & $(0.21$ & - & $0.32)$ & $\begin{array}{l}< \\
0.001\end{array}$ & 0.20 & $(0.14$ & - & $0.27)$ & $\begin{array}{l}< \\
0.001\end{array}$ \\
\hline \multicolumn{16}{|l|}{ Dietary pattern } \\
\hline Dietary diversity & 0.89 & $(0.80$ & - & $0.99)$ & 0.049 & 0.89 & $(0.77$ & - & 1.01) & 0.078 & 0.92 & $(0.75$ & - & 1.12) & 0.406 \\
\hline $\begin{array}{l}\text { Low fruit, vegetable, and dairy product } \\
\text { frequency }\end{array}$ & 1.02 & $(0.92$ & - & 1.13) & 0.723 & 1.02 & $(0.90$ & - & $1.17)$ & 0.728 & 1.01 & $(0.84$ & - & $1.22)$ & 0.922 \\
\hline High meat frequency & 1.18 & (1.07 & - & 1.32) & 0.002 & 1.19 & $(1.04$ & - & 1.36) & 0.010 & 1.18 & $(0.98$ & - & 1.41) & 0.079 \\
\hline Economic satisfaction & 1.04 & $(0.89$ & - & $1.21)$ & 0.620 & 1.05 & $(0.87$ & - & 1.26) & 0.624 & 0.98 & $(0.74$ & - & 1.29) & 0.869 \\
\hline Salaried employee (ref: No) & 0.88 & $(0.67$ & 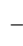 & 1.15) & 0.349 & 0.91 & $(0.67$ & - & 1.25) & 0.570 & 0.83 & $(0.49$ & - & 1.41) & 0.489 \\
\hline No hospitalization over the past year (ref: Yes) & 0.81 & $(0.58$ & - & 1.12) & 0.194 & 0.80 & $(0.53$ & - & 1.21) & 0.287 & 0.85 & $(0.50$ & - & 1.44) & 0.541 \\
\hline Subjective health & 0.90 & $(0.72$ & - & 1.12) & 0.353 & 0.98 & $(0.75$ & - & $1.28)$ & 0.868 & 0.73 & $(0.49$ & - & 1.09) & 0.128 \\
\hline Life satisfaction & 1.03 & $(0.81$ & - & $1.31)$ & 0.818 & 1.09 & $(0.80$ & - & 1.47) & 0.598 & 0.95 & $(0.63$ & - & 1.44) & 0.804 \\
\hline \multicolumn{16}{|l|}{ BMI categories (ref: $\mathrm{BMI}<18.5$ ) } \\
\hline $18.5 \leq \mathrm{BMl}<25.0$ & 0.51 & $(0.31$ & - & $0.84)$ & 0.008 & 0.50 & $(0.25$ & - & $0.99)$ & 0.049 & 0.55 & $(0.27$ & - & 1.13) & 0.101 \\
\hline $25.0 \leq \mathrm{BMI}<30.0$ & 0.64 & $(0.37$ & - & 1.10) & 0.103 & 0.65 & $(0.31$ & - & 1.36) & 0.255 & 0.61 & $(0.26$ & - & 1.40) & 0.244 \\
\hline $30.0 \leq \mathrm{BMl}$ & 0.67 & $(0.24$ & - & 1.86) & 0.446 & 1.30 & $(0.36$ & - & 4.65) & 0.686 & 0.11 & $(0.01$ & - & 1.10) & 0.060 \\
\hline \multicolumn{16}{|c|}{ Exercise frequency, enjoyment \& fulfillment (E\&F) (ref: $\leq 2$ times/week) } \\
\hline$\geq 3$ times/week, E\&F & 0.84 & $(0.62$ & - & 1.13) & 0.238 & 1.06 & $(0.72$ & - & 1.54) & 0.774 & 0.54 & $(0.33$ & - & $0.91)$ & 0.020 \\
\hline$\geq 3$ times/week & 0.92 & $(0.57$ & - & 1.48) & 0.715 & 1.05 & $(0.56$ & - & 1.95) & 0.888 & 0.74 & $(0.34$ & - & 1.61) & 0.449 \\
\hline$\leq 2$ times/week, E\&F & 0.81 & $(0.58$ & - & 1.11) & 0.191 & 0.90 & $(0.60$ & - & 1.35) & 0.609 & 0.64 & $(0.37$ & - & $1.12)$ & 0.120 \\
\hline No fall fractures in the past year (ref: Yes) & 0.62 & $(0.45$ & - & $0.84)$ & 0.002 & 0.61 & $(0.41$ & - & $0.92)$ & 0.017 & 0.61 & $(0.37$ & - & 1.02) & 0.057 \\
\hline No cigarette smoking status & 0.97 & $(0.78$ & - & 1.20) & 0.762 & 0.85 & $(0.66$ & - & 1.09) & 0.197 & 1.34 & $(0.88$ & - & 2.03) & 0.168 \\
\hline $\begin{array}{l}\text { Community and volunteer activity } \\
\text { engagement(ref: Sometimes or No) }\end{array}$ & 0.66 & $(0.53$ & 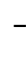 & $0.82)$ & $\begin{array}{l}< \\
0.001\end{array}$ & 0.62 & $(0.47$ & - & $0.81)$ & $<001$ & 0.77 & $(0.53$ & - & 1.12) & 0.167 \\
\hline Sex (ref: Men) & 0.56 & $(0.43$ & - & $0.73)$ & $\begin{array}{l}< \\
0.001\end{array}$ & 0.54 & $(0.38$ & - & $0.75)$ & $\begin{array}{l}<.001 \\
0.001\end{array}$ & 0.60 & $(0.37$ & - & $0.96)$ & 0.031 \\
\hline Age (+ 1 years old) & 1.03 & (1.01 & - & 1.05) & 0.004 & 1.00 & $(0.95$ & - & 1.05) & 0.947 & 1.02 & $(0.96$ & - & 1.08) & 0.582 \\
\hline
\end{tabular}

OR: odds ratio, $\mathrm{Cl}$ : confidence interval, ref: reference

independence 3 years later. Because principal components derived from the PCA will be uncorrelated, they are a good fit for use as independent variables for logistic regression analysis [23].

After adjusting for other dietary patterns or IADLrelated factors, the relationship between dietary diversity and decline in independence after 3 years became insignificant in both age stages. Simultaneously, high meat frequency was associated with a higher risk of decline in independence. Notably, later-stage older women showed the highest association with the risk of IADL disability. Consistent with results from other dietary pattern studies of older people [13], this study suggests that unhealthy dietary patterns, such as high meat intake, might impact the decline in independence on later-stage older people than rich dietary patterns of dietary diversity.
Diets with a high intake of animal protein, such as meats rich in saturated fatty acids, and a low intake of bluefish, rich in n-3 fatty acids, and soy-based foods with vegetable protein may promote a decline in independence. Meat may play a key role in the relationship between inflammation and low independence in older people. The Dietary Inflammatory Index (DII) [26] was created by Shivappa et al. to score 45 types of foods and nutrients based on their inflammatory properties, according to a review of 1943 research papers. DII scores have been validated using inflammatory markers, such as blood C-reactive protein (CRP), to indicate chronic inflammation [26]. The DII score of saturated fatty acids, which are abundant in meat, was 0.373 . It was the highest possible score and was evaluated as the highest proinflammatory nutrient. 


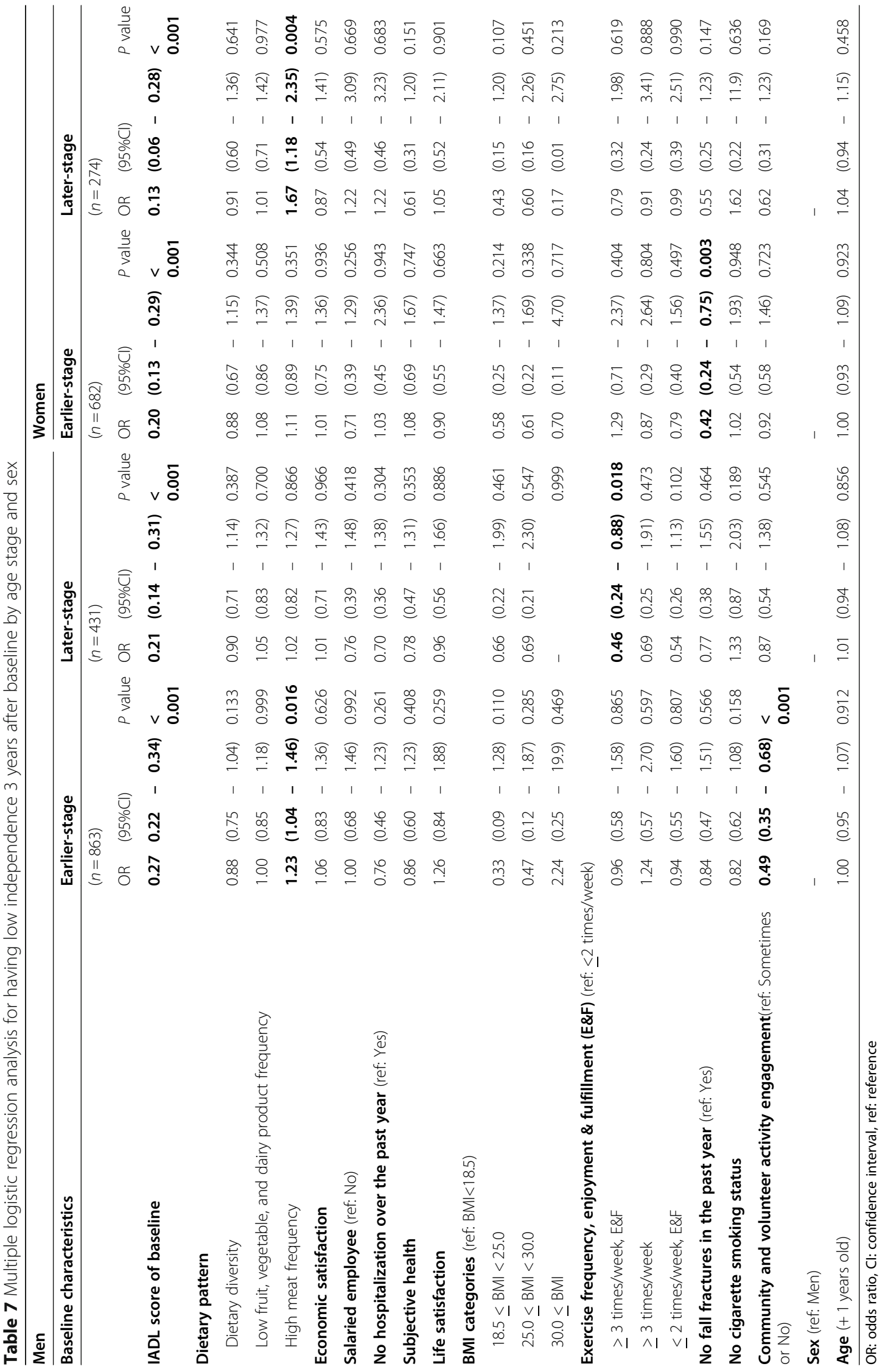


Table 8 Standardized effect by Multiple-Group Analysis and its difference between reference group.

\begin{tabular}{|c|c|c|c|c|c|c|c|c|c|c|c|c|c|}
\hline \multirow{2}{*}{\multicolumn{2}{|c|}{$\begin{array}{c}\text { Baseline } \\
\text { characteristics }^{a}\end{array}$}} & $\begin{array}{l}\text { IADL score of } \\
\text { baseline }\end{array}$ & \multicolumn{3}{|c|}{$\begin{array}{l}\text { High meat } \\
\text { frequency }\end{array}$} & \multicolumn{2}{|c|}{$\begin{array}{c}\text { BMI } \\
\text { categories }\end{array}$} & \multicolumn{2}{|c|}{$E \& F$} & \multicolumn{2}{|c|}{$\begin{array}{l}\text { No fall fractures in } \\
\text { the past year }\end{array}$} & \multicolumn{2}{|c|}{$\begin{array}{l}\text { Community and } \\
\text { volunteer activity } \\
\text { engagement }\end{array}$} \\
\hline & & S.E. ${ }^{b} \quad C R^{c}$ & S.E. ${ }^{b}$ & $C R$ & & S.E. ${ }^{b}$ & $C R^{c}$ & S.E. ${ }^{b}$ & $C R^{c}$ & S.E. ${ }^{b}$ & $C R^{c}$ & S.E. ${ }^{b}$ & $C R^{c}$ \\
\hline \multicolumn{14}{|c|}{ Age stage difference } \\
\hline \multicolumn{2}{|c|}{ Earlier-stage } & -0.45 & 0.05 & ref & & 0.03 & ref & 0.03 & ref 7 & * -0.05 & ref & -0.07 & ref \\
\hline \multicolumn{2}{|c|}{ Later-stage } & $-0.46-0.84$ & 0.06 & 0.07 & & -0.03 & -1.70 & -0.05 & 2.01 & -0.07 & 0.41 & -0.04 & 0.83 \\
\hline \multicolumn{14}{|c|}{ Sex difference } \\
\hline \multicolumn{2}{|l|}{ Men } & -0.45 & 0.05 & ref & & 0.03 & ref & -0.03 & ref & -0.03 & ref & -0.10 & ref 7 . \\
\hline \multicolumn{2}{|c|}{ Women } & $-0.41-1.53$ & 0.07 & 0.36 & & -0.03 & -1.55 & 0.02 & 1.49 & -0.12 & -1.83 & -0.03 & 2.09 \\
\hline \multicolumn{14}{|c|}{ Age stage and sex difference } \\
\hline \multirow{2}{*}{ Men } & Earlier-stage & -0.44 & 0.08 & 1.26 & & 0.06 & ref & 0.00 & -1.48 & -0.02 & -1.60 & -0.13 & ref $\supset *$ \\
\hline & Later-stage & $-0.46-1.16$ & 0.00 & 2.29 & - & -0.03 & -1.73 & -0.08 & ref- & -0.03 & -1.07 & -0.02 & 1.98 \\
\hline \multirow{2}{*}{ Women } & Earlier-stage & $-0.41-0.76$ & 0.04 & 2.40 & & ${ }^{* *}-0.02$ & -1.65 & 0.05 & 2.38 & ${ }^{* *}-0.11$ & ref & -0.01 & 3.02 \\
\hline & Later-stage & $-0.43-1.27$ & 0.17 & ref & **] & -0.05 & -1.83 & -0.04 & 0.71 & -0.13 & 1.19 & -0.08 & 1.00 \\
\hline
\end{tabular}

Conversely, the n-3 fatty acid content in bluefish was 0.436. Using the National Health and Nutrition Survey Data of 2572 Japanese adults, a positive association was found between DII scores and CRP. The higher the DII score, the higher the likelihood of observing high meat intake in Japanese adults [27]. Many studies have reported that a high-pro-inflammatory diet with high DII scores in older people is associated with disability or death [28].

In recent years, high protein intake by older people has been recommended as a support for the decline in independence prevention. The recommendation of eating moderate amounts of meat and other food varieties is necessary, especially for later-stage older people, and should be included in nutrition education.

\section{Comprehensive analysis of low independence after 3 years}

We carried out a comprehensive analysis by all participants and by age stages. By comparing those results, we clarified each age group's characteristics that are not revealed by all participants' results. Furthermore, those differences among age stage or sex were confirmed statistically.

In this study, an appropriate BMI was associated with preventing a decline in earlier-stage older men's independence. Earlier-stage older people were between 65 and 75 years of age, suggesting the necessity of continuing measures, such as weight management, to prevent chronic lifestyle diseases and aggravation. The high frequency of saturated fatty acid-rich meat in the earlierstage may be associated with the risk of decline in independence mediated by obesity-related lifestyle-related diseases. Simultaneously, in the later-stage, it was expected that decline could be more strongly affected by inflammation risk.

Furthermore, social participation, such as community and volunteer activity engagement, could prevent a disability in earlier-stage older people, especially for men. The use of resources other than specialists has also been reported [29]. For example, utilizing earlier-stage older people as leaders in volunteer activities in an independence decline prevention program for the latter group may present a model of support in the future.

Our results for the later-stage older people suggest that exercise frequency and E\&F may be priority support items for preventing a decline in independence after 3 years. A previous study of people aged 85 years and older reported that, even if the effective aerobic exercise consisting of 3 times per week was effective for older people's physical function after 6 months, it did not have a significant effect on their mental health [30]. The vulnerability of mental and psychological factors in older people is a multifaceted problem associated with declining independence. This study suggests that mental and psychological factors are essential needs, especially for later-stage older people.

Social participation might be one of the target factors that lead to the realization of successful aging to meet older people's needs for mental and physical health [14]. Our study also showed that "community and volunteer activity engagement" appeared to prevent low independence after 3 years in all age stages for both sexes. Because social support and social cohesion mediate the relationship between social participation and older people's health [14], older people who have more chances for social participation are more likely to receive support from community care, resulting in more physical activities [31]. 


\section{Limitations}

This study had several limitations. First, we evaluated the respondents' independence using the validated TMIG-IC, which asked for subjective information on the participants' ability to engage in IADL. Utilizing a numerical index as an outcome, such as healthy life expectancy, which has been calculated by a period of not needing nursing or support [32], would have provided effective evidence for making a strategy for health care support.

Next, dietary patterns were derived based on the frequency of food consumption. Although the BMI related to total intake was added to the multivariate-adjusted model analysis, it was not calculated based on a quantitative intake of multiple single food items. Furthermore, there are different approaches for assessing dietary patterns, namely, investigator-defined patterns methods, for example, "DASH" or "Mediterranean diet scores." Future studies should examine these practical methods further.

\section{Conclusions}

The present study was conducted for the communitydwelling independent older people in Japan, analyzing earlier and later-stage older people in both sexes. The results showed that the IADL disability in older people aged 75 and over was a rapid change and revealed that the characteristics associated with the risk of decline in independence 3 years later were statistically different among age stage and sex. It is necessary to implement community-based comprehensive supportive strategies to prevent disability in older people for long life globally, targeting age stages and sex separately.

\section{Abbreviations \\ BMI: BODY mass index; Cl: Confidence interval; CR: Critical ratio; DASH: Dietary approaches to stop hypertension; IADL: Instrumental activities of daily living; ICOPE: Integrated care for older people; n-3: Omega-3; n- 6: Omega-6; OR: Odds ratio; SEM: Structural Equation Modeling; S.E.: Standardized estimates; TMIG-IC: Tokyo Metropolitan Institute of Gerontology Index of Competence; FSDSC: Foundation of Social Development for Senior Citizens}

\section{Acknowledgments}

We would like to thank the staff of the FSDSC, especially Kazuki Yamato and Kiyoyuki Yakushiji, the participants who cooperated with the survey, and Editage (www.editage.com) for English language editing.

\section{Authors' contributions}

TH designed and conducted the survey and prepared raw data. TH, SuK, and SaK maintained, analyzed, and interpreted the data. SaK was a major contributor in writing the manuscript. All authors read and approved the final manuscript.

\section{Funding}

This study was partially funded by the FSDSC, which was used to conduct surveys and prepare the data. The funding body had the role of recruiting the participants and collecting questionnaires. They had no specific role in analysis and interpretation or manuscript conception and writing. SaK was funded by Sagami Women's University to analyze, edit, and publish this manuscript. The funding body had no role in the study design, data collection, analysis, interpretation, or manuscript conception and writing.

\section{Availability of data and materials}

The data that support the findings of this study are available from the Tokyo Metropolitan University. However, restrictions apply to the data availability, used under the current study's license, and are not publicly available. Nevertheless, data are available from the authors upon reasonable request and permission from the Tokyo Metropolitan University.

\section{Declarations}

Ethics approval and consent to participate

The Ethics Review Committee for Research on Humans at Sagami Women's University approved this study (No. 1793, October 27, 2017). The questionnaire was anonymized (ID numbering) to protect personal information. Written informed consent was obtained from all the study participants.

\section{Consent for publication}

Not applicable.

\section{Competing interests}

The authors declare no competing interests.

\section{Author details}

${ }^{1}$ Department of Food and Nutrition Science, Sagami Women's Junior College, 2-1-1 Bunkyo, Minami-ku, Sagamihara-shi, Kanagawa-ken 252-0383, Japan. ${ }^{2} T o k y o$ Metropolitan University, 1-1 Minami-Osawa, Hachioji-shi, Tokyo 192-0397, Japan. Department of Nursing, Seitoku University, 550 Iwase, Matsudo-shi, Chiba-ken 271-8555, Japan.

Received: 12 May 2020 Accepted: 7 June 2021

Published online: 26 June 2021

\section{References}

1. Cabinet Office Japan. Annual report on the ageing society [summary] FY 2019. 2019. https://www8.cao.go.jp/kourei/english/annualreport/2019/pdf/2 019.pdf. Accessed May 3, 2021.

2. Naruse T, Sakai M, Matsumoto H, Nagata S. Diseases that precede disability among latter-stage elderly individuals in Japan. BioScience Trends. 2015;9(4): 270-4. https://doi.org/10.5582/bst.2015.01059.

3. Ministry of Health, Labour and Welfare. Health insurance, an outline of the Japanese Medical System. https://www.mhlw.go.jp/bunya/iryouhoken/ iryouhoken01/dl/01_eng.pdf. Accessed May 3, 2021.

4. Arai $H$, Ouchi $Y$, Toba $K$, Endo $T$, Shimokado $K$, Tsubota $K$, et al. Japan as the front-runner of super-aged societies: perspectives from medicine and medical care in Japan. Geriatr Gerontol Int. 2015;15(6):673-87. https://doi. org/10.1111/ggi.12450.

5. Ministry of Health, Labour and Welfare. Guidelines on public health service for latter-stage older people. 2nd ed; 2019. https://www.mhlw.go.jp/ content/12401000/000604327.pdf. Accessed May 3, 2021

6. World Health Organization (WHO). Integrated care for older people: guidelines on community-level interventions to manage declines in intrinsic capacity. 2017. https://apps.who.int/iris/bitstream/handle/10665/258981/ 9789241550109-eng.pdf; jsessionid=694ABF8BE92676D3B678908298522A2 8 ? sequence $=1$. Accessed May 3, 2021.

7. McNaughton SA. Chapter 13. Dietary patterns. In: Marriott BP, Birt DF, Stallings VA, Yates AA, editors. Present knowledge in nutrition Vol. 2: clinical and applied topics in nutrition book. 11th ed: Elsevier Inc; 2020. p. 235-48.

8. Brink A, Brolsma E, Berendsen A, Rest O. The Mediterranean, dietary approaches to stop hypertension (DASH), and Mediterranean-DASH intervention for neurodegenerative delay (MIND) diets are associated with less cognitive decline and a lower risk of Alzheimer's disease-a review. Adv Nutr. 2019;10(6):1040-65. https://doi.org/10.1093/advances/nmz054.

9. Rest O, Berendsen A, Haveman-Nies A, Groot L. Dietary patterns, cognitive decline, and dementia: a systematic review. Adv Nutr. 2015;6(2):154-68. https://doi.org/10.3945/an.114.007617.

10. Mitchell K, Peeters A, Anstey K. Role of dietary pattern analysis in determining cognitive status in elderly Australian adults. Nutrients. 2015;7(2): 1052-67. https://doi.org/10.3390/nu7021052.

11. Zhang S, Tomata Y, Sugawara Y, Tsuduki T, Tsuji I. The Japanese dietary pattern is associated with longer disability-free survival time in the general 
elderly population in the Ohsaki cohort 2006 study. J Nutr. 2019;149(7): 1245-51. https://doi.org/10.1093/jn/nxz051.

12. D'Amico D, Parrott MD, Greenwood CE, Ferland G, Gaudreau P, Belleville S, et al. Sex differences in the relationship between dietary pattern adherence and cognitive function among older adults: findings from the NuAge study. Nutr J. 2020;19(1):58. https://doi.org/10.1186/s12937-020-00575-3.

13. Gu Q, Sable CM, Brooks-Wilson A, Murphy RA. Dietary patterns in the healthy oldest old in the healthy aging study and the Canadian longitudinal study of aging: a cohort study. BMC Geriatr. 2020;20(1):106. https://doi.org/1 0.1186/s12877-020-01507-w.

14. Douglas H, Georgiou A, Westbrook J. Social participation as an indicator of successful aging: an overview of concepts and their associations with health, vol. 41; 2017. p. 455-62.

15. Hoshi T. SES, environmental condition, three health-related dimensions, and healthy life expectancy. In: Hoshi T, Kodama S, editors. The structure of healthy life determinants: lessons from the Japanese aging cohort studies. Singapore: Springer; 2018. p. 175-92. https://doi.org/10.1007/978-981-10-662 9-0_11.

16. Kodama S, Kurimori S, Hoshi T, Taira K, Urasaki T, Ojiri Y, et al. Structural effects of cognitive factors and dietary diversity on self-rated health among healthy elderly in an Okinawan farm village. J Jpn Soc Nutr Food Sci. 2016; 69(4):151-62. (in Japanese). https://doi.org/10.4327/jsnfs.69.151.

17. Thorpe MG, Milte CM, Crawford D, McNaughton SA. Education and lifestyle predict change in dietary patterns and diet quality of adults 55 years and over. Nutr J. 2019;18(1):67. https://doi.org/10.1186/s12937-019-0495-6.

18. Kodama S, Kurimori S, Yamashiro K, Yakushiji K, Hoshi T. Association between subjective well-being in exercise frequency and independence three years later in community-dwelling, independent, elderly individuals. J Health Welfare Stat. 2019;66(6):1-8 (in Japanese).

19. Koyano W, Shibata H, Nakazato K, Haga H, Suyama Y. Measurement of competence: reliability and validity of the TMIG index of competence. Arch Gerontol Geriatr. 1991;13(2):103-16. https://doi.org/10.1016/0167-4943(91 )90053-S.

20. Fujiwara Y, Shinkai S, Amano H, Watanabe S, Kumagai S, Takabayashi K, et al. Test-retest variation in the Tokyo metropolitan Institute of Gerontology Index of competence in community-dwelling older people independent in daily living toward individual assessment of functional capacity. Jpn J Public Health. 2003;50(4):360-7 (in Japanese).

21. Ministry of Health, Labour and Welfare. Questionnaire for the elderly. (In Japanese) https://www.mhlw.go.jp/content/12401000/000580246.pdf. Accessed May 3, 2021.

22. Fujiwara Y, Kobayashi E, Fukaya T, Nishi M, Saito M, Nonaka K, et al. Crosssectional relationships between social determinants of health: annual income or subjective economic status and psychological variables. Jpn J Geriatr Psychiatry. 2012;23:211-20 (in Japanese).

23. Chaman Lal Sabharwal CL. Anjum B. data reduction and regression using principal component analysis in qualitative spatial reasoning and health informatics. Polibits. 2016;53:31-42. https://doi.org/10.17562/PB-53-3.

24. Kiyoshige E, Kabayama M, Gondo Y, Masui Y, Inagaki H, Ogawa M, et al. Age group differences in association between IADL decline and depressive symptoms in community-dwelling elderly. BMC Geriatr. 2019;19(1):309. https://doi.org/10.1186/s12877-019-1333-6.

25. Byrne BM. The hypothesized multigroup model. In: Structural equation modeling with Amos: basic concepts, applications, and programming. 3rd ed. New York: Routledge/Taylor \& Francis; 2016. p. 271-86. https://doi.org/1 $0.4324 / 9781315757421$

26. Shivappa N, Steck SE, Hurley TG, Hussey JR, Hébert JR. Designing and developing a literature-derived, population-based dietary inflammatory index. Public Health Nutr. 2014;17(8):1689-96. https://doi.org/10.1017/S13 68980013002115.

27. Yang Y, Hozawa A, Kogure M, Narita A, Hirata T, Nakamura T, et al. Dietary inflammatory index positively associated with high-sensitivity C-reactive protein level in Japanese from NIPPON DATA 2010. J Epidemiol. 2020;30(2): 98-107. https://doi.org/10.2188/jea.JE20180156

28. Tomata Y, Shivappa N, Zhang S, Nurrika D, Tanji F, Sugawara Y, et al. Dietary inflammatory index and disability-free survival in community-dwelling older adults. Nutrients. 2018;10(12):E1896.

29. Luger E, Dorner TE, Haider S, Kapan A, Lackinger C, Schindler K. Effects of a home-based and volunteer-administered physical training, nutritional, and social support program on malnutrition and frailty in older persons: a randomized controlled trial. J Am Med Dir Assoc. 2016;17(7):671.e9-671.e16.
30. Cho C, Han C, Sung M, Lee C, Kim M, Ogawa Y, et al. Six-month lower limb aerobic exercise improves physical function in young-old, old-old, and oldest-old adults. Tohoku J Exp Med. 2017;242(4):251-7. https://doi.org/10.1 620/tjem.242.251.

31. Smith GL, Banting L, Eime R, O'Sullivan G, van Uffelen JGZ. The association between social support and physical activity in older adults: a systematic review. Int J Behav Nutr Phys Act. 2017;4:56.

32. Kurimori S, Fukuda Y, Hoshi T, Takahashi A, Sunou K, Ota H. Utilization of the calculation results of national and 47 prefectural disability-adjusted life expectancy (DALE) and weighted disability prevalence (WDP) based on certified for long-term care using the calculation program. J Health Welfare Stat. 2017;64(3):26-33 (in Japanese).

\section{Publisher's Note}

Springer Nature remains neutral with regard to jurisdictional claims in published maps and institutional affiliations.
Ready to submit your research? Choose BMC and benefit from:

- fast, convenient online submission

- thorough peer review by experienced researchers in your field

- rapid publication on acceptance

- support for research data, including large and complex data types

- gold Open Access which fosters wider collaboration and increased citations

- maximum visibility for your research: over $100 \mathrm{M}$ website views per year

At BMC, research is always in progress.

Learn more biomedcentral.com/submissions 